\title{
Development of Energy Efficiency Estimation Method for Variable Refrigerant Flow Air-Conditioning System with Unbalanced Heat Load Operation
}

\author{
Masato Miyata $^{1}$, Koji Kurotori ${ }^{2}$, Napoleon Enteria ${ }^{3}$, Hideki Yamaguchi ${ }^{1}$, \\ Takao Sawachi ${ }^{4}$, Yasuo Kuwasawa ${ }^{1}$ \\ ${ }^{1}$ National Institute for Land and Infrastructure Management, Tsukuba, Japan \\ ${ }^{2}$ Centre for Better Living, Tsukuba, Japan \\ ${ }^{3}$ Mindanao State University, Iligan City, Philippines \\ ${ }^{4}$ Building Research Institute, Tsukuba, Japan
}

\begin{abstract}
This research proposes an appropriate method of estimating the energy efficiency of a Variable Refrigerant Flow air-conditioning system. In particular, this research tries to develop a method of accurately estimating energy efficiency when the heat load of each unit is unequal and unbalanced. This report presents the results of 36 performance measurements from a special laboratory that connects multiple constant temperature and humidity chambers. The experimental results indicated that the energy efficiency of the system when the load of each indoor unit was equal was higher than the efficiency when the load of each indoor unit was unbalanced, and the difference in efficiency was about $20 \%$. Based on the results, a mathematical model to estimate system energy efficiency taking into account the unbalanced load condition was developed.
\end{abstract}

\section{Introduction}

The number of buildings that have the VRF airconditioning system is increasing, but there are still many unclear points about actual performance, in particular, with regard to performance under a partial load condition (Goetzel, 2007; Watanabe, 2007). It is important to understand partial load performance and set reliable performance characteristics when performing a building energy simulation because it will have a significant influence on annual energy consumption of the system (Horie, 2012).

The previous study analysed the measurement data of actual buildings to clarify the efficiency under a partial load condition and showed that there was a variation of efficiency even at the same load ratio as shown in Figure 1 (Enteria, 2016a; Enteria, 2016b). Figure 1 also shows the performance curve assumed by the Japanese Building Energy Standard. This curve is expressed by the following formula (Building Energy Standard of Japan, 2013).

$$
\eta_{\text {ratio }}=\frac{1}{0.7823 L^{2}+0.0398 L+0.1779}
$$

Where:

$$
\begin{array}{ll}
\eta_{\text {ratio }} & : \text { COP ratio } \\
L & : \text { heat load ratio }
\end{array}
$$

The cause of this variation was assumed to be mainly due to bias (unbalance) in the heat amount handled by multiple indoor units. The efficiency tended to be higher when the heat amount of all indoor units of a system were equal, which was the test condition specified by ISO 15042:2017, JIS B 8615-1:1999, and JIS B 8616:2015, but efficiency tended to be lower when the heat amount handled by the indoor units was highly unbalanced.

This point was generally not considered but was ignored in existing building energy simulations. Performance curves assumed in many building system simulations, including EnergyPlus, were based on test results based on ISO 15042: 2017 (Tianzhen, 2016). The test results were based on the assumption that the cooling and heating load of each indoor unit was equal. However, in actual building operation, the load of each indoor unit was not even. For example, when air conditioning the interior zone and the perimeter zone with the same outdoor unit, a large difference might occur in the heat load of each indoor unit. In the case where the load of each indoor unit was unbalanced, even if the total amount of generated heat was the same, the refrigeration cycle changed depending on the degree of load unevenness and the power consumption of the compressor was different. Estimating energy consumption with ideal efficiency without considering unbalanced load operation might possibly mislead equipment designers and national energy policies.

This report clarified the influence of the unbalanced heat load to the system energy efficiency. Fundamental data on the energy efficiency of the VRF air-conditioning system was measured in an experimental facility that connected multiple constant temperature and humidity chambers.

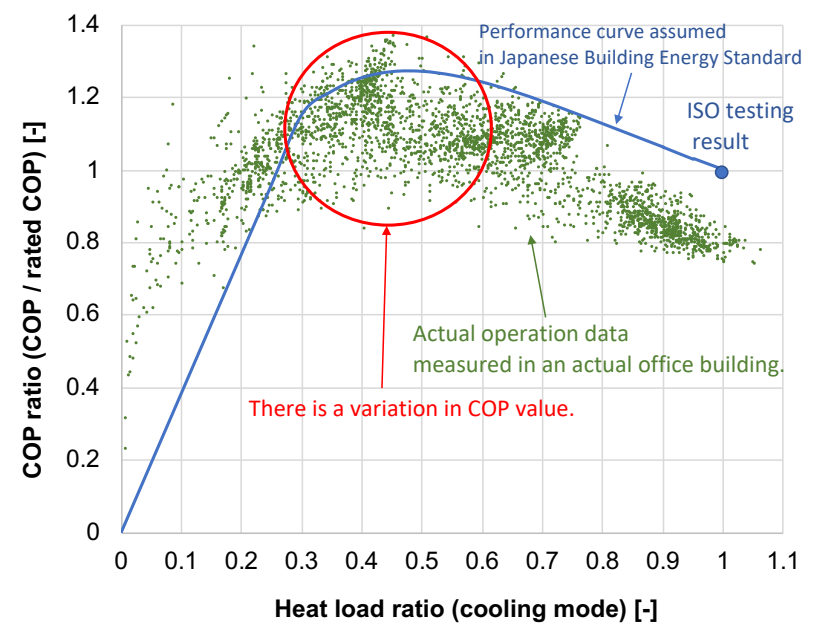

Figure 1: Performance characteristic of a VRF air-conditioning system. 

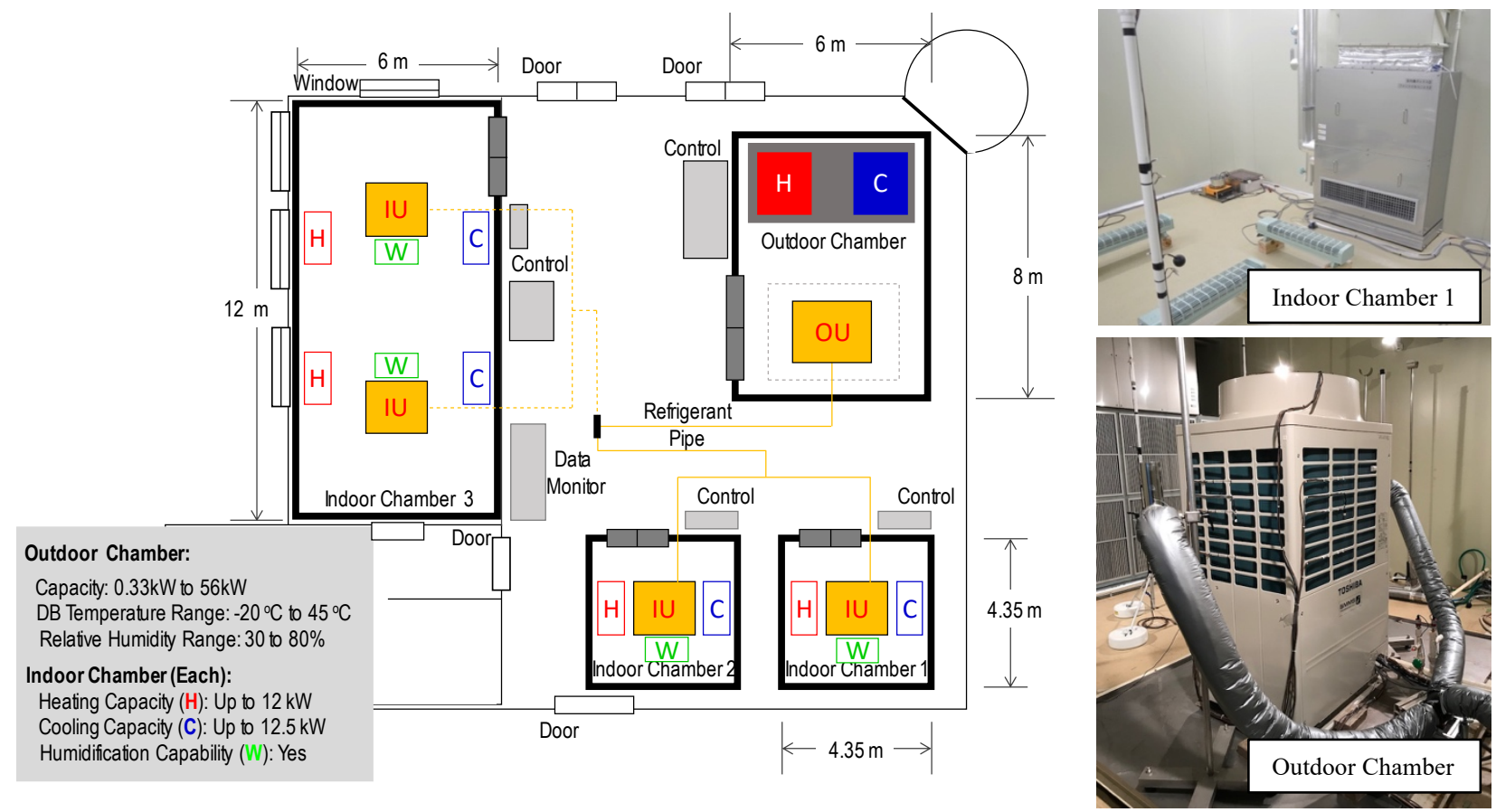

Figure 2: Outline of the testing facilities (located in Building Research Institute Japan).

\section{Test Facilities}

Figure 2 shows the technical diagram of the testing facilities used to make the performance evaluation of the VRF air-conditioning system. Figure 2 also shows the actual view of the testing facilities. The testing facilities consisted of one outdoor chamber and three indoor chambers. The outdoor chamber could support the system with an outdoor capacity of from $0.33 \mathrm{~kW}$ to $56 \mathrm{~kW}$. The outdoor chamber dry bulb (DB) temperature could vary from $-20^{\circ} \mathrm{C}$ to $45^{\circ} \mathrm{C}$. The relative humidity range could vary from $30 \%$ to $80 \%$, controlled by using a wet bulb temperature sensor for the outdoor temperature when it was above the freezing point of water. The three indoor chambers could simulate both cooling and heating loads. The two smaller indoor chambers (Indoor Chamber $1 \&$ 2) could support a heating load of up to $12 \mathrm{~kW}$ with humidification capability while the bigger indoor chamber (Indoor Chamber 3) could support a heating load of up to $24 \mathrm{~kW}$ with humidification capability. Both indoor chambers 1 and 2 could support a cooling load of up to $12.5 \mathrm{~kW}$, and indoor chamber 3 could support a cooling load of up to $25 \mathrm{~kW}$; the two indoor units could be installed in tandem for the case of indoor chamber 3 . Control and monitoring devices were installed to gather the needed parameters.

\section{Test Specimen}

The air conditioner to be tested in this research was an air conditioner made by Toshiba Carrier Corporation (SMMS-i series) whose rated heating capacity was 22.4 $\mathrm{kW}$ (8 HP), rated heating COP was 4.24 and cooling COP was 3.70 . Four $5.6 \mathrm{~kW}$ indoor units were connected (two for indoor chambers No. 1 and No. 2 and the other two for indoor chamber No. 3).

Since the purpose of this research was to reveal the actual performance assumed to be installed in actual buildings, the rotation speed of the compressors and the expansion valve opening degrees were not fixed intentionally though it was usual in the case of the testing specified by ISO 15042:2017.

\section{Measurement}

The heat load amount of each indoor unit was estimated by measuring the air enthalpy difference of the indoor unit. The total heat load amount of the air-conditioning system was obtained as the sum of the heat load amount of all indoor units. The inlet and outlet air temperature and humidity of the indoor unit were measured with temperature and humidity sensors, and the air volume of the indoor unit was estimated by measured fan rotation speeds. In addition, in order to measure the refrigerant pressure, pressure sensors were installed in the outdoor unit, and watt meters were installed in order to measure the energy consumption of the indoor units and the outdoor unit. The sampling time of the energy consumption was set to 1 second, and the sampling time of the others was set to 5 seconds.

\section{Experimental Cases and Methods}

Table 1 shows the experimental cases carried out. The experiment for the heating mode was conducted from September 2017 to February 2018. The experiment for the cooling mode was conducted from August 2018 to December 2018. As shown in Table 1, the test cases were classified into the following three groups:

- High load range cases (Case-H series, the total heat load ratio was $75 \%$ or more),

- Medium load range cases (Case-M series, the total heat load ratio was about $50 \%$ ),

- Low load range cases (Case-L series, the total heat load ratio was $25 \%$ or less). 
Table 1a: Experimental cases carried out

(heating mode).

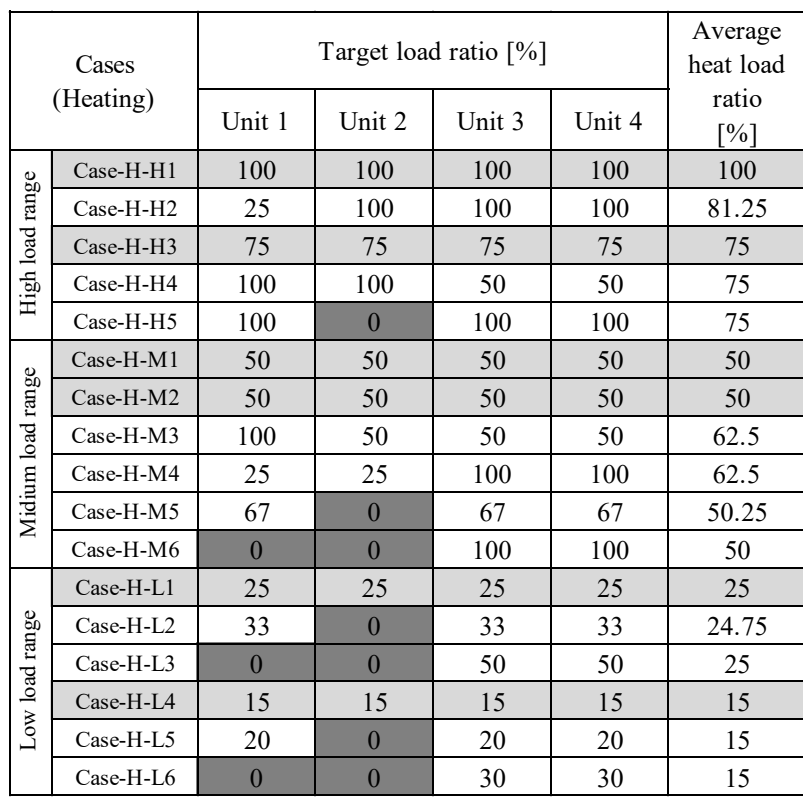

The following are cases where the heat load ratios of all the indoor units were the same and there was no unbalanced load condition.

- For heating mode, Case-H-H1, Case-H-H3, Case-HM1, Case- H-M2, Case-H-L1 and Case-H-L4.

- For cooling mode, Case-C-H1a, Case-C-H1b, CaseC-H3, Case-C-M1, Case-C-M2, Case-C-L1 and Case-C-L4.

In all other cases, the heat load ratio of each indoor unit was not the same; it was unbalanced. Case-H-M1 and Case-H-M2 or Case-C-M1 and Case-C-M2 were exactly the same conditions. The purpose of the same two experiments was to confirm the reproducibility of this experiment.

In heating mode testing, the outdoor chamber was maintained at a dry-bulb temperature of $7^{\circ} \mathrm{C}$ and a wetbulb temperature of $6^{\circ} \mathrm{C}$. The set point of the indoor room air temperature was $20^{\circ} \mathrm{C}$. The air volume setting was maximum. In cooling mode testing, the outdoor chamber was maintained at a dry-bulb temperature of $35^{\circ} \mathrm{C}$ and a wet-bulb temperature of $29^{\circ} \mathrm{C}$. The set point of the indoor room air temperature was $27^{\circ} \mathrm{C}$. The air volume setting was maximum.

For each case, the output of the load generators (fan coil units for heating mode, electric heaters for cooling mode) installed in the indoor chambers was adjusted so that the heat load ratio of each indoor unit was maintained at the target load ratio shown in Table 1. Because this experimental facility did not have a function to stably and strictly control the heat load ratio of the indoor unit, the load ratio shown in Table 1 was just a target value, and the actual achieved load ratio was somewhat different from the target value.
Table 1b: Experimental cases carried out

(cooling mode).

\begin{tabular}{|c|c|c|c|c|c|c|}
\hline \multirow{2}{*}{\multicolumn{2}{|c|}{$\begin{array}{c}\text { Cases } \\
\text { (Cooling) }\end{array}$}} & \multicolumn{4}{|c|}{ Target load ratio [\%] } & \multirow{2}{*}{$\begin{array}{c}\text { Average } \\
\text { heat load } \\
\text { ratio } \\
{[\%]}\end{array}$} \\
\hline & & Unit 1 & Unit 2 & Unit 3 & Unit 4 & \\
\hline \multirow{6}{*}{ 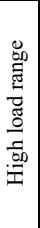 } & Case-C-H1a & 100 & 100 & 100 & 100 & 100 \\
\hline & Case-C-H1b & 100 & 100 & 100 & 100 & 100 \\
\hline & Case-C-H2 & 25 & 100 & 100 & 100 & 81.25 \\
\hline & Case-C-H3 & 75 & 75 & 75 & 75 & 75 \\
\hline & Case-C-H4 & 100 & 100 & 50 & 50 & 75 \\
\hline & Case-C-H5 & 100 & 0 & 100 & 100 & 75 \\
\hline \multirow{7}{*}{ 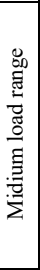 } & Case-C-M1 & 50 & 50 & 50 & 50 & 50 \\
\hline & Case-C-M2 & 50 & 50 & 50 & 50 & 50 \\
\hline & Case-C-M3 & 15 & 62 & 62 & 62 & 50.25 \\
\hline & Case-C-M4 & 25 & 25 & 75 & 75 & 50 \\
\hline & Case-C-M5 & 15 & 15 & 85 & 85 & 50 \\
\hline & Case-C-M6 & 75 & 0 & 62 & 62 & 49.75 \\
\hline & Case-C-M7 & 100 & 100 & 0 & 0 & 50 \\
\hline \multirow{6}{*}{ 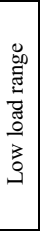 } & Case-C-L1 & 25 & 25 & 25 & 25 & 25 \\
\hline & Case-C-L2 & 33 & 0 & 33 & 33 & 24.75 \\
\hline & Case-C-L3 & 0 & 0 & 50 & 50 & 25 \\
\hline & Case-C-L4 & 15 & 15 & 15 & 15 & 15 \\
\hline & Case-C-L5 & 20 & 0 & 20 & 20 & 15 \\
\hline & Case-C-L6 & 0 & 0 & 30 & 30 & 15 \\
\hline
\end{tabular}

\section{Data Analysis}

With respect to the experimental data of each case, the data where the heat load ratio and the outlet air temperature of each indoor unit were close to the target values and stable was selected, and the data for one hour during this stable period was averaged. Among the data for this one hour, the state in which the air conditioner intermittently repeatedly turned on and off (intermittent operation) was also included. Although there was still room for discussion on how to extract this one-hour data, the graph was checked visually, and the data of the time span judged to be stable was extracted arbitrarily. As an example, the experimental data for Case- $\mathrm{H}-\mathrm{H} 1$ is shown in Figure 3. For Case-H-H1, operational data from 11:58 to $12: 58$ was extracted as stable data.

\section{Analysis of Performance}

For each case, the average heat load amount of the outdoor unit (the sum of the heat load amount of the indoor units) and the average energy consumption were calculated using the measured data. Using these two values, the average operation efficiency of the system could be calculated as follows:

$$
\eta=\frac{Q_{\text {ave }}}{E_{\text {ave }}}
$$

Where:
$\eta \quad$ : Operation efficiency [-]
$Q_{\text {ave }} \quad$ : Average heat load amount $[\mathrm{kW}]$
$E_{\text {ave }} \quad$ : Average energy consumption $[\mathrm{kW}]$ 
Table 2 shows the results of the experiment for the heating mode and Figure 4 shows the relationship between the average heat load amount of the outdoor unit and the average operation efficiency. The heat load of outdoor unit is calculated by adding the heat load of the indoor units shown in Table 2. For Case-H-H4, Case-H-M3, and Case-H-M4, the room air temperature was significantly lower than the set value of $20^{\circ} \mathrm{C}$. Further studies are needed in order to know why these cases could not maintain the room air temperature at the set value.

The findings obtained from the experimental results of heating mode are shown below.

1) For Case-H-M1 and Case-H-M2, experiments with exactly the same conditions were conducted on different days with the purpose of confirming the reproducibility of the experiment. As shown in Table 2, the results of both cases were almost the same. In this experiment, intentional fixation of the compressor rotation speed and of the expansion valve opening degree was not conducted, but it was confirmed that there was to some extent the reproducibility.

2) Regarding the experimental results in high load range cases, when comparing Case-H-H3 (without load unbalanced) and Case-H-H5 (load ratio was $100 \%$ for three units and the other was stopped), it was clear that the efficiency of Case-H-H5 was about $15 \%$ lower even though the outdoor unit load was almost the same (about $16 \mathrm{~kW}$ ). When comparing Case-H-H3 and Case-H-H2 (load ratio was $100 \%$ for three units and $25 \%$ for one unit),
Case-H-H2 was also about $15 \%$ less efficient. Compared to Case-H-H3 in Case-H-H5 and Case-H$\mathrm{H} 2$, the refrigerant pressure of Case- $\mathrm{H}-\mathrm{H} 3$ and Case$\mathrm{H}-\mathrm{H} 5$ was higher as shown in Table 2, which seemed to cause performance deterioration.

3) Regarding the experimental results in the medium load range cases, Case-H-M1 (without load unbalanced) and Case-H-M3 (load ratio was 50\% for 3 three units and $50 \%$ for 1one unit) have nearly the same operation efficiency, but Case-H-M5 (load ratio was $67 \%$ for 3 units and the other was stopped) was about $8 \%$ less efficient than Case-H-M1. Furthermore, Case-H-M6 (load ratio was $100 \%$ for 2 units and the others were stopped) was about $20 \%$ less efficient.

4) Regarding the experimental results in the low load range cases, Case-H-L2 (load ratio was 33\% for 3 three units and the other was stopped) and Case-HL3 (load ratio was $50 \%$ for 2 units and the others were stopped) were more efficient than Case-HL1(without load unbalanced). This was because Case-H-L1 intermittently repeatedly turned on and off for a total load factor of $25 \%$, which caused an energy loss, but Case-H-L2 and Case-H-L3 were continuously operating.

Table 3 and Figure 5 show the results of the experiment for cooling mode. For Case-C-H2 and Case-C-H4, the room air temperature was significantly higher than the set value of $27^{\circ} \mathrm{C}$. Further studies are needed in order to know why these cases could maintain the room air temperature at the set value.
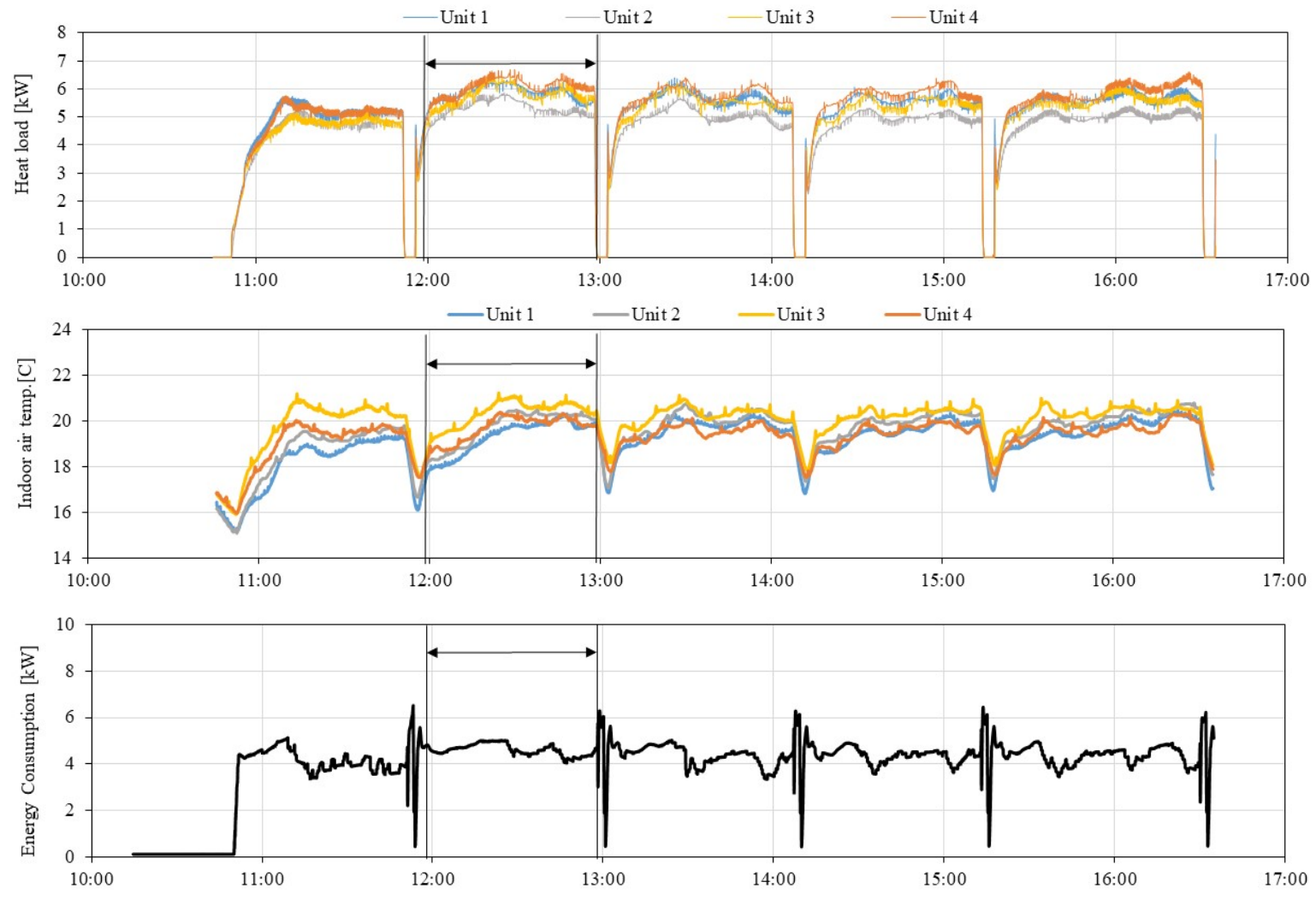

Figure 3: An example of measured data (Case-H-H1). 
Table 2: Experimental results for all cases (heating mode).

\begin{tabular}{|c|c|c|c|c|c|c|c|c|c|c|c|c|c|c|}
\hline & \multirow{2}{*}{$\begin{array}{c}\text { Cases } \\
\text { (Heating) }\end{array}$} & \multicolumn{3}{|c|}{ Outdoor unit } & \multicolumn{4}{|c|}{$\begin{array}{l}\text { Actual achived heat load } \\
\text { of indoor units }[\mathrm{kW}]\end{array}$} & \multicolumn{4}{|c|}{ Indoor temperature $\left[{ }^{\circ} \mathrm{C}\right]$} & \multicolumn{2}{|c|}{$\begin{array}{c}\text { Refrigerent pressure } \\
{[\mathrm{MPa}]}\end{array}$} \\
\hline & & Heat load & Energy & Operation & & & & & & & & & & \\
\hline \multirow{5}{*}{ 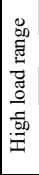 } & Case-H-H1 & 22.81 & 4.62 & 4.94 & 5.83 & 5.26 & 5.72 & 6.00 & 19.27 & 19.71 & 20.33 & 19.68 & 2.33 & 1.80 \\
\hline & Case-H-H2 & 18.53 & 3.86 & 4.81 & 0.98 & 5.28 & 6.01 & 6.27 & 21.77 & 19.72 & 19.10 & 18.67 & 2.33 & 1.79 \\
\hline & Case-H-H3 & 16.47 & 2.92 & 5.64 & 4.17 & 3.82 & 4.03 & 4.45 & 19.73 & 19.82 & 19.95 & 20.38 & 2.11 & 1.64 \\
\hline & Case-H-H4 & 16.13 & 2.77 & 5.82 & 5.42 & 5.02 & 2.71 & 2.98 & 14.73 & 15.10 & 20.86 & 20.74 & 2.01 & 1.53 \\
\hline & Case-H-H5 & 16.13 & 3.36 & 4.80 & 5.38 & - & 5.81 & 4.94 & 20.19 & - & 19.80 & 20.13 & 2.32 & 1.82 \\
\hline \multirow{6}{*}{ 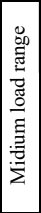 } & Case-H-M1 & 11.00 & 1.98 & 5.56 & 2.78 & 2.52 & 2.99 & 2.71 & 20.49 & 19.97 & 20.44 & 19.86 & 2.01 & 1.55 \\
\hline & Case-H-M2 & 11.42 & 2.03 & 5.63 & 2.75 & 2.69 & 2.79 & 3.18 & 20.95 & 20.34 & 21.40 & 21.26 & 2.07 & 1.55 \\
\hline & Case-H-M3 & 12.32 & 2.13 & 5.78 & 5.07 & 1.87 & 2.77 & 2.61 & 14.24 & 20.56 & 20.52 & 20.32 & 1.96 & 1.50 \\
\hline & Case-H-M4 & 11.11 & 2.13 & 5.22 & 1.03 & 0.76 & 4.58 & 4.74 & 21.21 & 21.18 & 16.10 & 15.74 & 2.13 & 1.63 \\
\hline & Case-H-M5 & 11.73 & 2.30 & 5.10 & 3.93 & - & 4.10 & 3.70 & 19.17 & - & 20.26 & 19.94 & 2.17 & 1.67 \\
\hline & Case-H-M6 & 11.65 & 2.59 & 4.50 & - & - & 5.60 & 6.05 & - & - & 19.95 & 19.38 & 2.36 & 1.82 \\
\hline \multirow{6}{*}{ 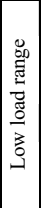 } & Case-H-L1 & 5.47 & 1.57 & 3.48 & 1.42 & 1.04 & 1.22 & 1.79 & 20.36 & 20.43 & 21.34 & 21.27 & 2.02 & 1.75 \\
\hline & Case-H-L2 & 5.77 & 1.52 & 3.80 & 2.03 & - & 1.48 & 2.26 & 20.01 & - & 21.34 & 21.14 & 2.06 & 1.82 \\
\hline & Case-H-L3 & 5.75 & 1.45 & 3.97 & - & - & 2.42 & 3.33 & - & - & 20.82 & 20.53 & 2.13 & 1.82 \\
\hline & Case-H-L4 & 3.45 & 1.23 & 2.80 & 0.96 & 0.83 & 0.85 & 0.76 & 20.65 & 21.23 & 21.15 & 21.07 & 1.89 & 1.82 \\
\hline & Case-H-L5 & 3.64 & 1.36 & 2.68 & 0.94 & - & 1.58 & 1.12 & 20.70 & - & 21.15 & 21.21 & 2.00 & 1.91 \\
\hline & Case-H-L6 & 3.09 & 1.51 & 2.05 & - & - & 1.45 & 1.64 & - & - & 21.50 & 21.35 & 2.05 & 1.89 \\
\hline
\end{tabular}

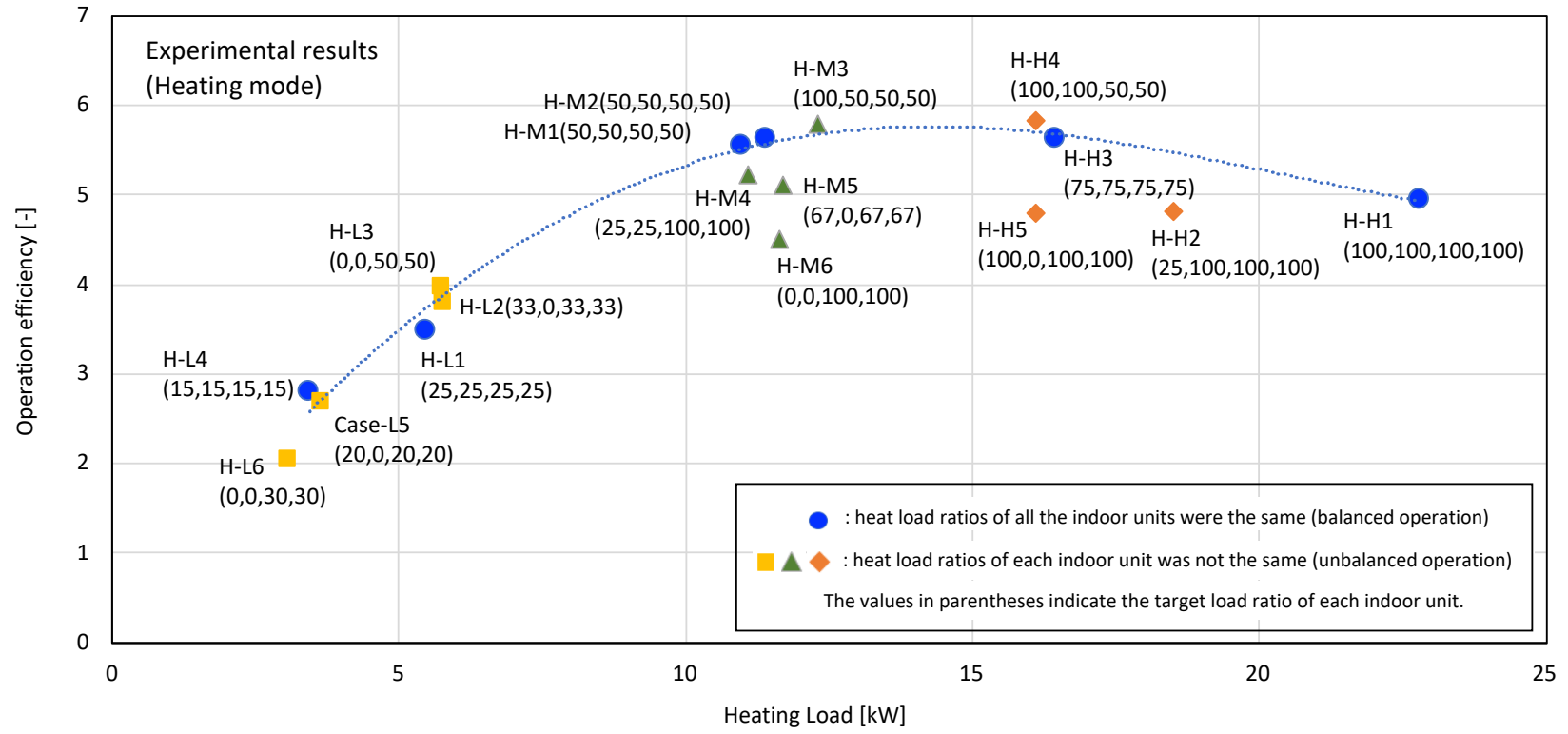

Figure 4: Experimental results plotted on the performance curve (heating mode).

The findings obtained from the experimental results of cooling mode are shown below. Experimental data in cooling mode showed almost the same trend as experimental data in heating mode.

1) For Case-C-M1 and Case-C-M2, experiments with exactly the same conditions were conducted on different days. As shown in Table 3, the results of both cases were almost the same. It was also confirmed that there was to some extent reproducibility for cooling mode as well as heating mode.

2) Regarding the experimental results in the high load range cases, the efficiency of Case-C-H5 (load ratio was $100 \%$ for three units and the other was stopped) seemed to be lower than the expected value estimated using the results of the balanced condition test results (Case-C-H1a, Case-C-H1b and Case-C$\mathrm{H} 3$ ), which was drawn as a blue dotted line in Figure 5.

3) Regarding the experimental results in the medium load range cases, Case-C-M3 (load ratio was $62 \%$ for three units and $15 \%$ for one unit), Case-C-M4 (load ratio was $75 \%$ for two units and $25 \%$ for two units) and Case-C-M6 (load ratio was $75 \%$ for one unit and $62 \%$ for two units and the other was stopped) was about $7 \%$ less efficient than Case-CM1(without load unbalanced). Furthermore, CaseC-M7 (load ratio was $100 \%$ for two units and the others were stopped) was about $20 \%$ less efficient. 
Table 3: Experimental results for all cases (cooling mode).

\begin{tabular}{|c|c|c|c|c|c|c|c|c|c|c|c|c|c|c|}
\hline & \multirow{2}{*}{$\begin{array}{c}\text { Cases } \\
\text { (Cooling) }\end{array}$} & \multicolumn{3}{|c|}{ Outdoor unit } & \multicolumn{4}{|c|}{$\begin{array}{l}\text { Actual achived heat load } \\
\text { of indoor units }[\mathrm{kW}]\end{array}$} & \multicolumn{4}{|c|}{ Indoor temperature $\left[{ }^{\circ} \mathrm{C}\right]$} & \multicolumn{2}{|c|}{$\begin{array}{c}\text { Refrigerent pressure } \\
{[\mathrm{MPa}]}\end{array}$} \\
\hline & & $\begin{array}{l}\text { Heat load } \\
{[\mathrm{kW}]}\end{array}$ & $\begin{array}{c}\text { Energy } \\
\text { consumption } \\
{[\mathrm{kW}]}\end{array}$ & $\begin{array}{c}\text { Efficiency } \\
{[-]}\end{array}$ & Unit 1 & Unit 2 & Unit 3 & Unit 4 & Unit 1 & Unit 2 & Unit 3 & Unit 4 & High & Low \\
\hline \multirow{6}{*}{ 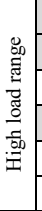 } & Case-C-H1a & 20.83 & 4.43 & 4.70 & 5.49 & 5.89 & 4.72 & 4.73 & 28.44 & 28.70 & 27.44 & 28.92 & 2.35 & 1.11 \\
\hline & Case-C-H1b & 24.19 & 6.24 & 3.87 & 6.02 & 6.34 & 5.76 & 6.08 & 27.03 & 27.49 & 27.17 & 28.58 & 2.37 & 1.02 \\
\hline & Case-C-H2 & 14.76 & 2.59 & 5.70 & 1.51 & 5.19 & 4.05 & 4.01 & 26.81 & 29.22 & 28.01 & 29.47 & 2.32 & 1.22 \\
\hline & Case-C-H3 & 18.02 & 3.37 & 5.35 & 4.70 & 4.88 & 4.41 & 4.03 & 27.69 & 27.42 & 28.03 & 28.35 & 2.25 & 1.15 \\
\hline & Case-C-H4 & 15.89 & 2.53 & 6.29 & 4.83 & 4.94 & 2.97 & 3.15 & 30.21 & 29.81 & 27.44 & 28.42 & 2.23 & 1.27 \\
\hline & Case-C-H5 & 14.29 & 2.72 & 5.26 & 5.13 & - & 4.60 & 4.57 & 28.56 & - & 27.75 & 29.21 & 2.24 & 1.13 \\
\hline \multirow{7}{*}{ 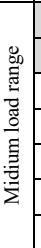 } & Case-C-M1 & 12.15 & 1.90 & 6.41 & 3.08 & 3.19 & 2.93 & 2.95 & 26.82 & 26.63 & 27.04 & 28.12 & 2.30 & 1.28 \\
\hline & Case-C-M2 & 12.19 & 1.94 & 6.27 & 3.01 & 3.22 & 3.03 & 2.93 & 26.82 & 26.84 & 27.17 & 28.04 & 2.31 & 1.27 \\
\hline & Case-C-M3 & 11.84 & 2.01 & 5.89 & 1.06 & 4.07 & 3.44 & 3.27 & 26.67 & 27.51 & 27.48 & 28.43 & 2.31 & 1.25 \\
\hline & Case-C-M4 & 10.70 & 1.84 & 5.81 & 1.68 & 1.83 & 3.60 & 3.59 & 27.06 & 27.77 & 27.63 & 28.92 & 2.33 & 1.26 \\
\hline & Case-C-M5 & 10.72 & 2.01 & 5.33 & 0.78 & 0.96 & 4.45 & 4.53 & 26.67 & 27.20 & 27.97 & 29.35 & 2.27 & 1.18 \\
\hline & Case-C-M6 & 11.27 & 1.95 & 5.78 & 4.12 & - & 3.53 & 3.61 & 28.30 & - & 27.13 & 28.29 & 2.28 & 1.23 \\
\hline & Case-C-M7 & 11.44 & 2.23 & 5.14 & 5.63 & 5.81 & - & - & 28.49 & 27.52 & - & - & 2.33 & 1.12 \\
\hline \multirow{6}{*}{ 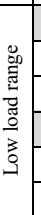 } & Case-C-L1 & 5.55 & 1.78 & 3.11 & 1.42 & 1.71 & 1.05 & 1.36 & 26.83 & 27.23 & 27.65 & 27.66 & 2.27 & 0.94 \\
\hline & Case-C-L2 & 4.72 & 1.56 & 3.03 & 1.79 & - & 1.31 & 1.62 & 26.72 & - & 27.56 & 27.49 & 2.27 & 0.98 \\
\hline & Case-C-L3 & 5.58 & 1.49 & 3.75 & - & - & 1.79 & 3.79 & - & - & 26.37 & 27.09 & 2.29 & 0.92 \\
\hline & Case-C-L4 & 5.35 & 1.72 & 3.11 & 1.23 & 1.53 & 0.85 & 1.73 & 26.57 & 27.02 & 27.19 & 27.12 & 2.25 & 0.98 \\
\hline & Case-C-L5 & 4.35 & 1.59 & 2.74 & 1.68 & - & 1.29 & 1.38 & 26.38 & - & 27.34 & 27.49 & 2.34 & 0.96 \\
\hline & Case-C-L6 & 3.47 & 0.91 & 3.83 & - & - & 1.66 & 1.81 & - & - & 26.93 & 27.20 & 2.20 & 1.08 \\
\hline
\end{tabular}

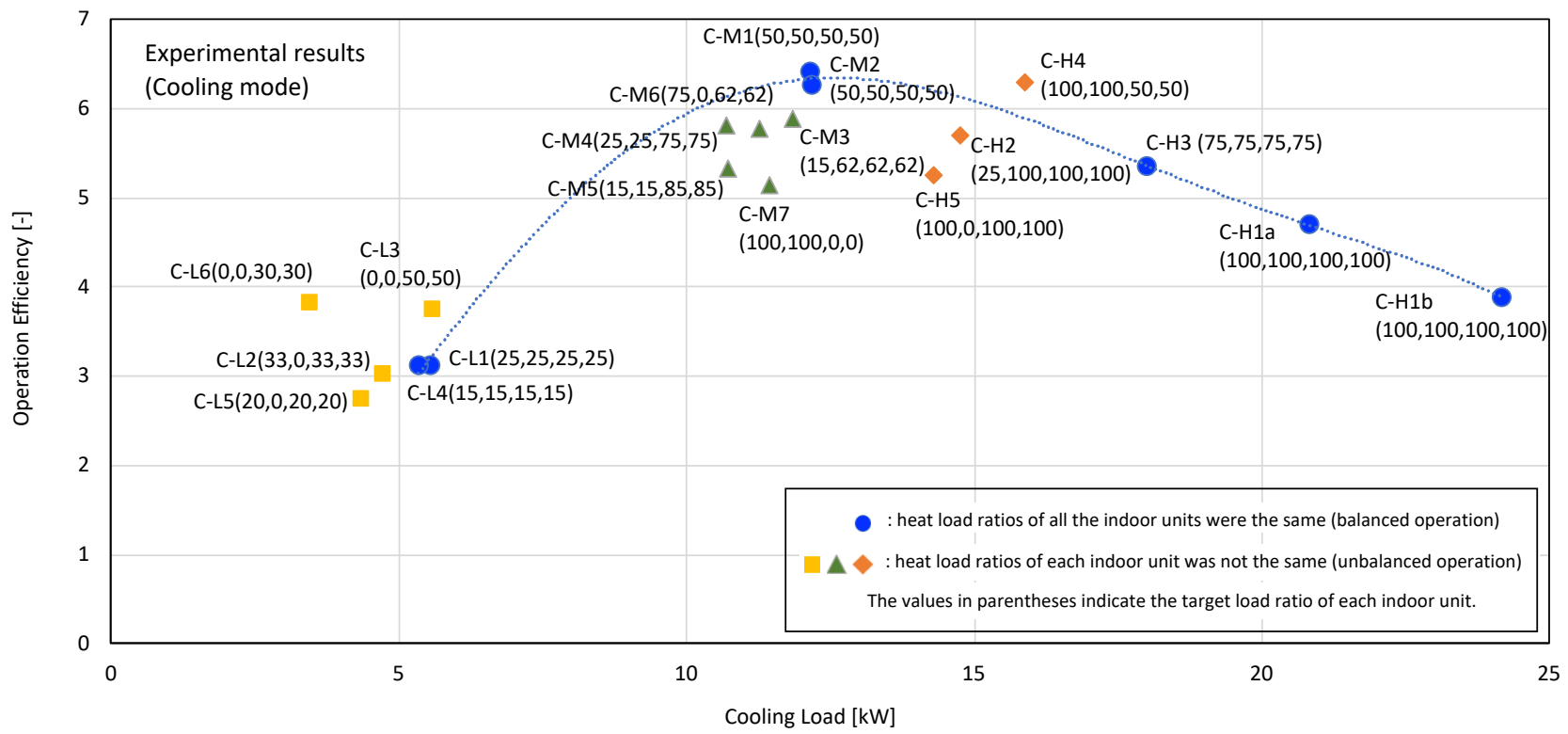

Figure 5: Experimental results plotted on the performance curve (cooling mode).

4) Regarding the experimental results in the low load range cases, as with the result of heating mode, Case-C-L3 (load ratio was 50\% for two units and the others were stopped) were more efficient than CaseH-L1(without load unbalanced).

\section{Estimation of Energy Performance}

Using the experimental results, a mathematical model to estimate the system energy efficiency taking into account the unbalanced load condition was developed. Here, because the experimental results showed that the efficiency change due to unbalanced load was small in the low load range and it was found that it was greatly affected by the ON-OFF operation, only the high load range and the medium load range were targeted in this paper.

The model equation proposed in this paper is shown below.

$$
\begin{aligned}
& C O P_{\text {heating }}=k_{h a} P_{\text {ref, }, \text { high }}+k_{h b} \\
& C O P_{\text {cooling }}=k_{c a} P_{\text {ref,low }}+k_{c b} \\
& P_{\text {ref, }, \text { high }}=k_{h c} L_{\text {max }}+k_{h d} \\
& P_{\text {ref,low }}=k_{c c} L_{\text {max }}+k_{c d} \\
& L_{\text {max }}=\max \left(L_{\text {unitl }}, L_{\text {unit }}, \ldots, L_{\text {unit } N}\right)
\end{aligned}
$$

Where:

$\mathrm{COP}_{\text {heating }}$ : Operation efficiency for heating mode [-] 


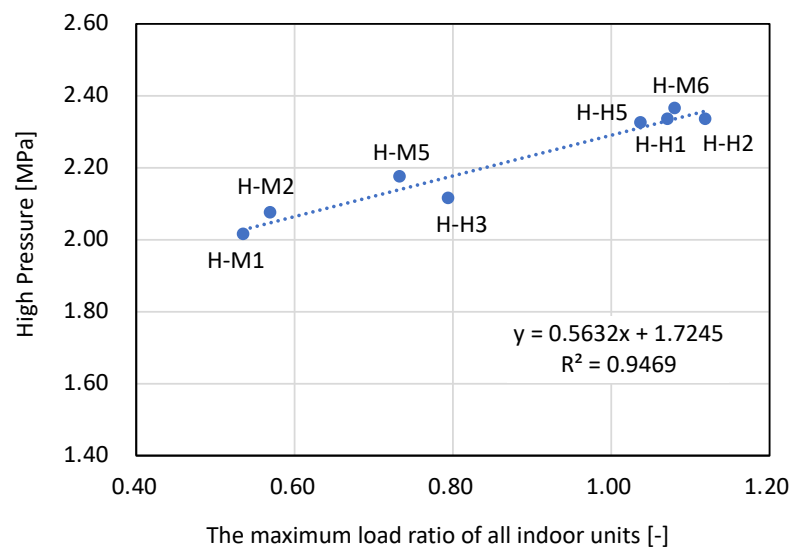

Figure 6: Relationship between $L_{\max }$ and $P_{\text {ref,high }}$ (heating mode).

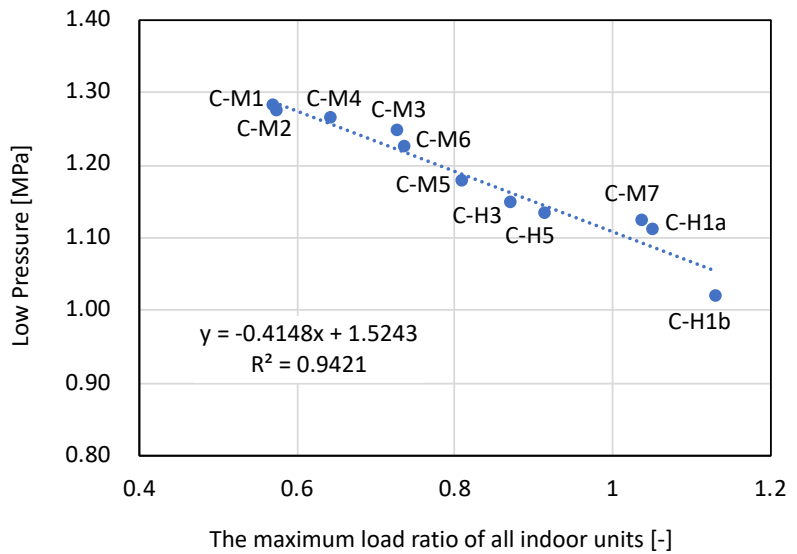

Figure 8: Relationship between $L_{\max }$ and $P_{\text {ref,low }}$ (cooling mode).

COP $P_{\text {cooling }}$ : Operation efficiency for cooling mode [-] $P_{\text {ref,high }}:$ Refrigerant pressure of high-pressure side [MPa]

$P_{\text {ref,low }}$ : Refrigerant pressure of low-pressure side [MPa]

$L_{\text {max }}$ : The maximum load ratio of all indoor units [-]

$L_{\text {unitN }}:$ Load ratio of indoor unit N [-]

$k$ : Model Parameters

Figures $6,7,8$, and 9 show the results of estimating the model parameters $k$ based on the results of the experiment. Figures 10 and 11 show the relationship between the COP estimated using the developed model and the actually measured COP. It is clear that the developed model can estimate the energy performance of unbalanced load condition with high accuracy.

\section{Discussion and Conclusion}

This paper showed the experimental results on the energy performance of the VRF air-conditioning system to clarify the influence of the unbalanced heat load of indoor units and developed a mathematical model to estimate

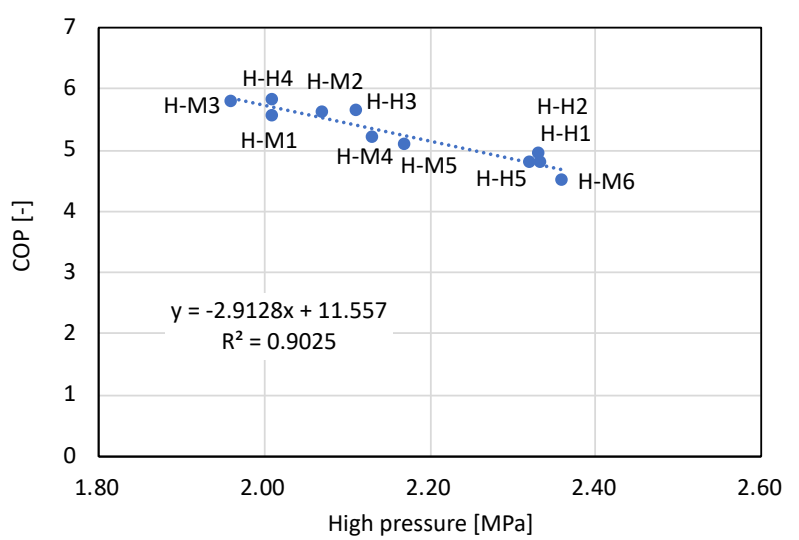

Figure 7: Relationship between $P_{\text {ref,high }}$ and $\mathrm{COP}_{\text {heating }}$ (heating mode).

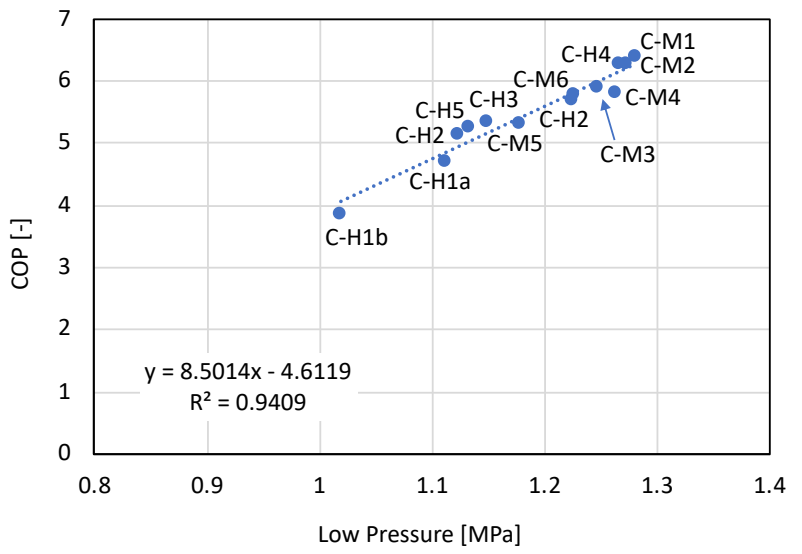

Figure 9: Relationship between $P_{\text {ref,low }}$ and $\mathrm{COP}_{\text {cooling }}$ (cooling mode).

system energy efficiency taking into account an unbalanced load condition. The testing method specified in ISO 15042:2017 was assumed to be conducted under the condition that the heat load amount of all the indoor units was equal, but in actual buildings, the heat load amount of all the indoor units was not equal for the following reasons:

a) Difference in heat load between the perimeter zone and the interior zone. Especially for buildings with poor thermal insulation performance and airtight performance, the difference may be larger.

b) Difference in heat load from appliances in the room (for example, there was a place where heat generation was locally higher because many copy machines were located in the place).

c) Difference in how the rooms were used (for example, the office room and the meeting room were airconditioned with the same outdoor unit).

The experimental results showed that the unbalanced load operation caused about a $20 \%$ energy loss at maximum. Because this paper clarified the trend via limited experiments only for limited air conditioners, more detailed experiments should be carried out in the future and clarify the mechanism by which the unbalanced heat 


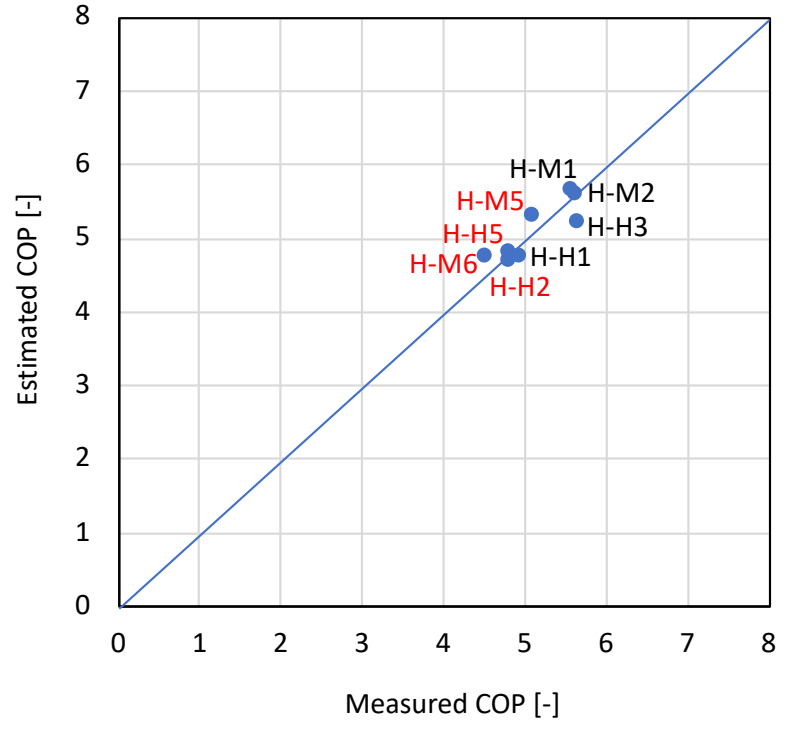

Figure 10: Verification results (heating mode).

load influences energy consumption efficiency. But this research revealed that it is necessary to incorporate this mechanism into building energy simulations in order to accurately calculate the annual energy consumption of buildings.

\section{Acknowledgement}

This work was supported by JSPS KAKENHI Grant Number JP17K18351.

\section{References}

Building Energy Standard of Japan (2013). Part 1. Nonresidential buildings. Method for calculations and judgments in conformity. Supervised by National Institute of Land and Infrastructure Management (NILIM) and Building Research Institute (BRI).

Doo Yong Park, Gyeong Yun, Kang Soo Kim (2017). Experimental evaluation and simulation of a variable refrigerant- flow (VRF) air-conditioning system with outdoor air processing unit, Energy and Buildings, Volume 146, 1, pp122-140

Enteria, N., Yamaguchi, H., Miyata, M., Sawachi, T. and Kuwasawa, Y. (2016). Performance evaluation of the variable refrigerant flow (VRF) air-conditioning system subjected to partial and unbalanced thermal loadings. JSME Journal of Thermal Science and Technology. 11, 1.

Enteria, N., Yamaguchi, H., Miyata, M., Sawachi, T. and Kuwasawa Y. (2016). Performance Evaluation of the Variable Refrigerant Flow (VRF) Air-Conditioning System Subjected to Partial Loadings at Different Outdoor Air Temperatures. JSME Journal of Thermal Science and Technology. 11, 2.

Goetzel, W., Variable refrigerant flow systems. ASHRAE Journal 49, 4 (2007).

Horie, H. and Hihara, E. (2012). Study on annual performance of room air conditioners under part load

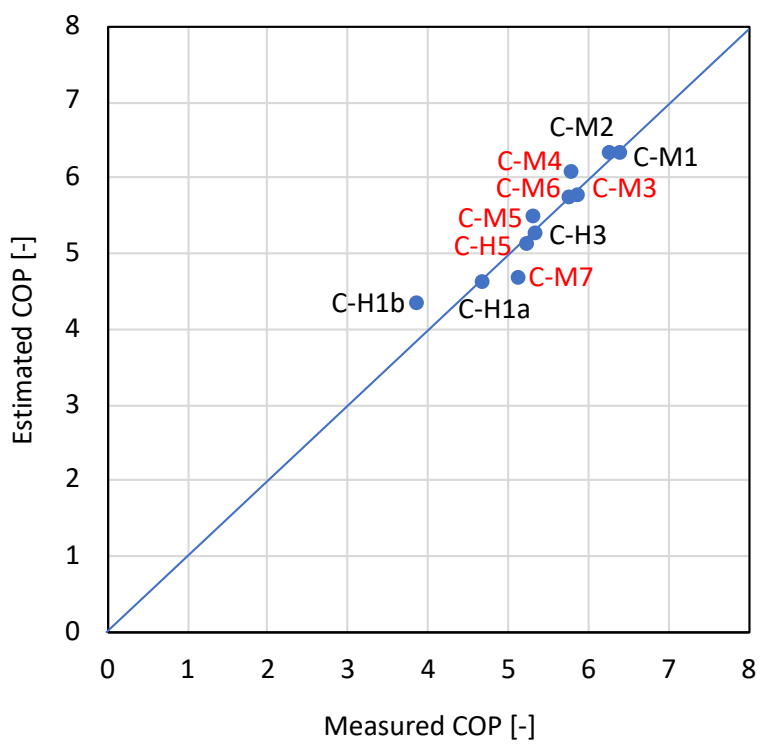

Figure 11: Verification results (cooling mode).

conditions. Proceedings of the International Refrigeration and Air Conditioning Conference.

Japan Industrial Standard. JIS B 8616:2015 (2015). Package air-conditioners. Japanese Standard Organization.

Japan Industrial Standard. JIS B 8615-1:1999 (1999). Ducted air-conditioners and air-to-air heat pumps testing and rating for performance. Japanese Standard Organization.

Tianzhen Hong, Kaiyu Sun, et al. (2016). Development and validation of a new variable refrigerant flow system model in EnergyPlus, Energy and Buildings, Volume 117, 1, 2016, pp. 399-411

Tolga N. Aynur (2010). Variable refrigerant flow systems, Energy and Buildings, Volume 42, Issue 7, July 2010, pp. 1106-1112

Watanabe, C., Nagamatsu, K., Nakayama, H., Hirota, M. and Ohashi, E. (2007). Performance characteristics of multi-type air-conditioners for building under partial thermal load operations. Proceedings of the International Symposium on EcoTopia Science. 